\title{
The role of response-produced cues in paired-associate transfer as a function of intralist stimulus similarity
}

DAVID M. del CASTILLO AND HENRY C. ELLIS UNIVERSITY OF NEW MEXICO

This experiment was designed to determine if the effects of acquired distinctiveness and equivalence pretraining depend upon the initial discriminability of the task stimuli. The Ss were assigned to one of eight experimental conditions, consisting of four types of stimulus pretraining and two levels of intralist stimulus similarity followed by a discriminative transfer task involving the same stimuli. Distinctiveness pretraining resulted in positive transfer, the amount of which was independent of the level of stimulus similarity. Equivalence pretraining did not result in any transfer at either level of stimulus similarity.

Dollard \& Miller (1950) have proposed that attaching response-produced cues to stimuli will affect the subsequent generalization of responses made to these stimuli. Studies designed to test this conception have frequently shown that attaching distinctive verbal labels to a set of similar stimuli (acquired distinctiveness of cues) facilitates the learning of new responses to those stimuli in a subsequent task, presumably because of reduced generalization among the task stimuli (e.g., Ellis \& Muller, 1964; Goss \& Greenfeld, 1958). In turn, attaching similar or common verbal labels to a set of stimuli (acquired equivalence of cues) interferes with the acquisition of new responses to those stimuli, presumably because of increased generalization among the task stimuli (e.g., Katz, 1963). More specifically, it has been suggested that the effect of attaching response-produced cues to a set of stimuli is a joint function of (a) the degree of intralist similarity of the set of stimuli, and (b) the distinctiveness of the response-produced cues (Goss, 1955).

The present experiment was designed to test these two assumptions. It was predicted that the facilitating effect of attaching distinctive verbal labels to a set of stimuli should be relatively greater when the stimuli are highly similar than when they are very low in similarity. Adding distinctive cues to a set of stimuli which are already very discriminable should have little facilitating effect. Further, it was predicted that the negative effect of attaching common mediating cues to a set of stimuli should be relatively greater when the stimuli are very dissimilar than when they are highly similar, since adding common cues to stimuli which are already very similar would have a negligible effect. Method

Ten volunteer Ss were assigned at random to one of eight conditions of the experiment, consisting of four types of stimulus pretraining and two levels of intralist stimulus similarity (high or low). Ss given labeling pretraining were given a paired-associate (PA) task in which they were required either to associate a different verbal label (two syllable, Haagen adjectives) to each of the four stimuli, Distinctiveness (D) pretraining; or to associate one verbal label to two of the stimuli, and a second label to the remaining two stimuli, Equivalence (E) pretraining. In the third pretraining condition, Ss were given Observation (O) pretraining in which they were required to "observe and discriminate" among the four stimuli of a set, but they were not required to attach any labels to them. The fourth condition involved no pretraining $(\mathrm{N})$, and Ss assigned to this condition began the transfer task without any prior practice with the stimuli. Following pretraining, Ss were immediately given a PA transfer-task which required that they learn to press a different switch to each of the four stimuli experienced during pretraining.

The two sets of stimuli were each composed of four circular patches of light which differed in intensity. The lights in the low-similarity set ranged from 1.00 to $.25 \mathrm{log} \mathrm{ft}-\mathrm{c}$ in steps of $.25 \mathrm{log} \mathrm{ft}-\mathrm{c}$, while those in the high-similarity set ranged from 1.00 to $.55 \mathrm{log}$ $\mathrm{ft}-\mathrm{c}$ in steps of $.15 \mathrm{log} \mathrm{ft}-\mathrm{c}$. Intensity was controlled by varying the voltage impressed across a $75 \mathrm{~W}$ projection bulb. The stimuli and labels were presented on a verbal discrimination device similar to that described by Goss \& Greenfeld (1958). The light intensities were presented on the lower of two circular screens on a stimulus panel, while the labels were presented on the upper screen. A response unit mounted below the stimulus panel contained four toggle switches arranged in a semicircle.

During pretraining, the PA list was presented at a $2: 2 \mathrm{sec}$ rate. Sis pretrained with the low-similarity stimuli were given 24 trials, while those pretrained with the high-similarity stimuli were given 36 trials in order to equate terminal performance. During the transfer task the stimuli were presented for $2 \mathrm{sec}$ on each of the 18 trials. A noncorrection procedure was used in which $S$ was allowed to select only one switch on each trial. If $\mathrm{S}$ made a correct response, a confirmation light was illuminated but not if S's response was incorrect.

\section{Results and Discussion}

Pretraining. Analysis of the mean total correct anticipations on the last four pretraining trials for the 


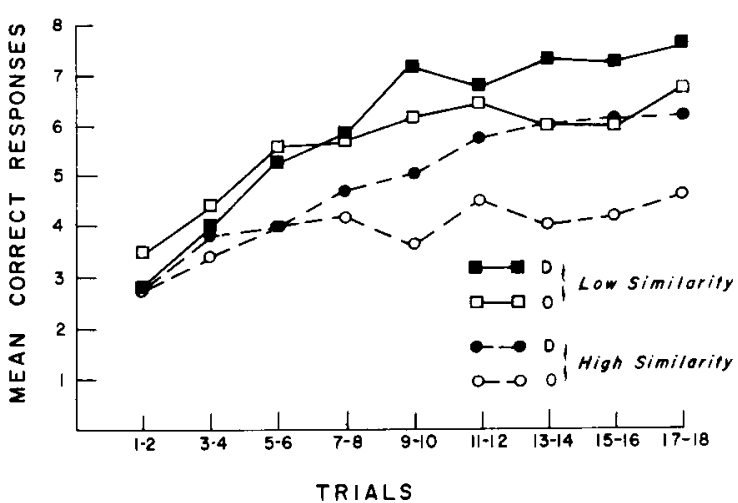

Fig. 1. Mean correct responses on discriminative transfer task as a function of trials, level of intralist stimulus similarity, and type of pretraining ( $D$ vs 0 ).

$D$ and $E$ pretraining conditions under high and low levels of intralist stimulus similarity indicated that Ss given E pretraining made significantly more correct anticipations on the last four trials than Ss given $D$ pretraining $(p<.01)$. This finding reflects the fact that Ss given $E$ pretraining had only two labels to learn rather than four labels as in $D$ pretraining. However, since no comparisons were made between the effects of $D$ and $E$ pretraining on transfer performance, this difference is unimportant and, therefore, requires no correction for chance responding. Analysis of the pretraining data also indicated that the terminal performance of $\mathrm{Ss}$ given $\mathrm{D}$ pretraining and those given $E$ pretraining did not differ as a function of level of intralist similarity $(p>.05)$.

Transfer performance. The data with regard to the predictions made from the acquired distinctiveness of cues hypothesis are shown in Fig. 1. From an inspection of these data it appears that the predicted interaction between type of stimulus pretraining and level of intralist stimulus similarity is present; however, an analysis of variance of these data fails to support this interpretation. Neither the interaction between pretraining and similarity, nor the interaction between pretraining, similarity, and trials on the transfer task achieved statistical significance ( $p>.05)$. In contrast, inspection of Fig. 1 shows that Ss given D pretraining made more correct responses than those given $O$ pretraining with identical stimuli at both high and low levels of intralist stimulus similarity, a result which is consistent with previous findings (Ellis \& Muller, 1964; Goss \& Greenfeld, 1958). Moreover, Ss made more correct responses when the stimuli were low in similarity than when they were high in similarity. These interpretations are supported by a significant main effect for pretraining $(p<.01)$, and for similarity $(p<.01)$. Comparisons between $D$ and $N$ pretraining conditions yielded results which parallel those for the $D$ vs $O$ comparisons. Thus, it is clear that with this range of differences in intralist stimulus similarity and type of pretraining, there was sufficient opportunity for differential positive transfer to occur. Nevertheless, the amount of transfer from $D$ pretraining compared with $O$ pretraining did not depend upon the level of intralist stimulus similarity. Underwood (1966, p. 521) has also attempted to demonstrate this interaction within an $A-B, A-D$ paradigm. Although the predicted interaction was present, the effect was much less than expected.

Turning now to the hypothesis of acquired equivalence of cues, it was predicted that E pretraining would result in negative transfer and that this effect would be relatively greater when the task stimuli were low in intralist similarity than when they were high in similarity. Analysis of the transfer task data for $E$ and $O$ conditions indicated that only the main effects for similarity and trials were statistically reliable $(p<.01)$. The E vs $\mathrm{N}$ comparisons yielded parallel results. Thus, no support was found for the acquired equivalence of cues hypothesis nor for the prediction that varying the similarity of the stimuli affects the magnitude of learned equivalence.

\section{References}

DOLlaRD, J., \& MILLER, N. E. Personality and psychotherapy.New York: MoGraw-Hill, 1950.

ELLIS, H. C., \& MULLER, D. G. Transfer of perceptual learning following stimulus predifferentiation. J. exp. Psychol, 1964, 68, 388-395.

GOSS, A. E. A stimulus-response analysis of the interaction of cueproducing and mediating responses. Psychol. Rev., 1955, 62, $20-31$.

GOSS, A. E., \& GREENFELD, N. Transfer to a motor task as influenced by conditions and degree of prior discrimination training. J. exp. Psychol., 1958, 55, 258-269.

KATZ, P. A. Effects of labels on children's perception and discrimination learning. J. exp. Psychol., 1963, 66, 423-428.

UNDERWOOD, B. J. Experimental psychology. New York: AppletonCentury-Crofts, 1966.

Note

1. This research was supported by National Science Foundation Grant GB-3432. 